\title{
GOBIERNO ABIERTO, PODER E INFLUENCIA: ANÁLISIS CRÍTICO A UNA PROPUESTA DE PRONTA PROPAGACIÓN ${ }^{*}$
}

\author{
Víctor S. Peña \\ El Colegio de Sonora, México \\ vpena@colson.edu.mx
}

\section{RESUMEN}

El gobierno abierto es un concepto que se ha propagado rápidamente en los gobiernos, la sociedad civil y la academia. El presente artículo analiza la evolución del concepto y la influencia que ha tenido en los últimos años. En la primera sección se describen algunas aproximaciones conceptuales del gobierno abierto que han sido llevadas a la práctica. Posteriormente, se hace analizan los esfuerzos realizados por el Instituto Nacional de Acceso a la Información Pública (INAI) de México para implementar el gobierno abierto en el nivel subnacional. Se destaca que en este proceso se realizaron ajustes al concepto de gobierno abierto utilizado por cada gobierno subnacional. En la tercera sección se abordan los desafíos que enfrentan los decisores de política cuando se enfrentan a un concepto (gobierno abierto) cuya definición sigue en elaboración. Finalmente, se presentan las conclusiones del estudio.

Palabras clave: Gobierno abierto, Implementación, Metáforas, México.

* Este artículo se elaboró en el marco del proyecto Transparencia y gobernanza en los gobiernos locales de México: Avances y retrocesos en la apertura de las instituciones públicas dentro del Programa de Apoyo a Proyectos de Investigación e Innovación Tecnológica (PAPIIT) de la Universidad Nacional Autónoma de México. Versiones similares de este texto se presentaron en: Congreso de la Asociación Mexicana de Ciencias Políticas y Bienal Territorios en Movimiento de la Universidad de Guanajuato (ambos en agosto de 2016), Congreso del CLAD (en noviembre de 2016). 


\title{
OPEN GOVERNMENT, POWER AND INFLUENCE: CRITICAL ANALYSIS ON A PROMPT PROPAGATION PROPOSAL
}

\begin{abstract}
Open government is a concept that has spread rapidly in governments, the civil society and the academic world. This article analyzes the development of the concept and the influence it has had in recent years. The first section presents a description of some conceptual approaches to open government that have been put into practice. Subsequently, there is a discussion on the efforts made by the Mexican Instituto Nacional de Acceso a la Información Pública (National Institute of Acces to Public Information) to implement open government at the subnational level. It is noted that, in this process, there were adjustments to the concept of open government used by each subnational government. The third section deals with the challenges faced by policy-makers when dealing with a concept (open government) whose definition is still under development. Finally, the conclusions of the study are presented.
\end{abstract}

Keywords: Open Government, Implementation, Metaphors, Mexico. 


\section{INTRODUCCIÓN}

El término gobierno abierto, más bien de manera selectiva que comprensiva, se ha propagado rápidamente dentro de las estructuras gubernamentales, el trabajo de la sociedad civil organizada y algunos textos académicos. Una de las muchas aristas que sobre el particular puede explorarse se conforma en los (presuntos) beneficios que trae (o traería) a los involucrados: mucho de lo hasta ahora escrito y dicho corre por esta senda; otra, la aquí considerada, pretende comprender cómo es que un término sin consistencia conceptual (con lo que implica pretender su implementación en estas condiciones) puede ser adoptado por gobiernos de distintos países.

A manera de elementos para la construcción de una primera hipótesis de trabajo, considero que: 1) el gobierno abierto es un término que interesa a los gobiernos antes que a ciudadanía, dado que su funcionamiento involucra la posibilidad de generar legitimidad frente a participantes; 2) el gobierno abierto interesa a un sector ciudadano que, sin perder sus matices críticos, existe y se fortalece cerca del gobierno; 3) el gobierno abierto es una agenda promovida y apoyada desde el exterior, con su origen inmediato en el gobierno estadounidense y su adopción, a manera de frente común, por países denominados democráticos; 4) la indefinición del gobierno abierto (que no es sinónimo a indeterminación conceptual) permite su adaptación, con el visto bueno de la coalición promotora internacional, según los actores involucrados en lo local. El presente trabajo pretende seguir estos elementos en una narrativa que considere la evolución del concepto y su análisis a la partir del poder y la influencia que ha tenido a lo largo de los años.

Considerando las hipótesis de trabajo, para el presente escrito se hace un recuento conceptual del gobierno abierto para la construcción de un marco básico para su entendimiento y para la obtención de una orientación empírica se analizó el ejercicio desarrollado por el Instituto Nacional de Acceso a la Información Pública (INAI) durante 2015 a nivel subnacional en México. De esta manera, el siguiente artículo tiene la siguiente estructura. En la primera sección se describen algunas aproximaciones conceptuales del gobierno abierto llevadas a la práctica. Posteriormente, se hace una relación de los ejercicios locales o subnacionales del INAI en la materia, destacando la evolución y ajustes del concepto utilizado. En el tercer apartado, se presentan las consideraciones de lo que implica, para el implementador, estar frente a una expresión cuya definición sigue en elaboración. Finalmente, se comparten las reflexiones que, hasta el momento, se han construido. 


\section{GOBIERNO(S) ABIERTO(S): SUS ESQUEMAS}

Un modelo es un punto de referencia, una composición que se busca imitar o reproducir; en una acepción menos pretensiosa, es una representación en pequeño de alguna cosa. Sin un concepto definitivo para el gobierno abierto, hablar de modelos parece inapropiado. Frente a esto, identificar esquemas -la representación gráfica o simbólica, el resumen de un discurso atendiendo sus caracteres más significativos-, es una alternativa ${ }^{1}$. Pues bien, ubicando al gobierno abierto es más sencillo identificar esquemas. Y de ahí, particularmente del análisis de cómo han funcionado, proponer líneas generales para una política pública que lo ubique como una práctica habitual.

\section{El gobierno abierto antes de la Alianza para el Gobierno ABIERTo}

¿Cuándo comenzó a utilizar la característica de la apertura como cualidad de lo gubernamental? Autores como Sandoval Almazán (2013) y Valenzuela (2014) han identificado antecedentes ubicados en la decada de los ańos cincuenta del siglo pasado. Por la proximidad temporal, un antecedente más inmediato se enncuentra en un white paper (reporte o guía, con el análisis de un asunto y, de ser posible, su referencia teórica) del gobierno londinense en 1993. Ahí se establece que "un gobierno abierto es parte de una democracia efectiva [...] [por lo que los] ciudadanos deben poder acceder y tener la información y el análisis sobre la cual la decisión gubernamental fue tomada" (Canciller del Ducado de Lancaster 1993. Traducción propia). Desde la arena académica, Stuart Weir y David Beetham (1999) dedicaron importantes apartados de su obra Political Power and Democratic Control in Britain a la apertura del gobierno. De acuerdo con ellos, uno de los cuatro componentes de la democracia representantiva es tener instituciones gubernmanetales permanentemente abiertas y rindiendo cuentas. Ellos lo llamaban un "open, accountable and responsive government” (Weir y Beetham 1999:10).

Si bien en el gobierno y en la academia pueden rastrearse antecedentes desde la década de los años noventa, en la cultura popular el término ha estado presente desde antes. Villoria Mendieta (2012) refiere su uso por Jim Hacker, personaje ficticio de la serie británica llamada Yes Minister, transmitida de 1986 a 1988 por la BBC: para cumplir con su programa electoral, Hacker mejoraría la transparencia a partir del open government.

1 Cuando la fuente consultada habla de modelo, se ha mantenido. 
De cualquier manera, es imposible determinar que alguno de estos precedentes hayan servido como base de lo que hoy conocemos bajo este título.

\section{GOBIERNO ABIERTO (LA PROPUESTA NORTEAMERICANA)}

El gobierno abierto, en su versión actual, viene directamente de la administración norteamericana de Barack Obama. Son tres los hitos identificables, los dos primeros definidos a partir de los documentos Transparencia y Gobierno Abierto de enero de 2009 y Directriz para el Gobierno Abierto de diciembre de ese mismo ańo. El tercero de ellos comienza en 2011, cuando el mandatario estadounidense presenta, en la Asamblea General de las Naciones Unidas, el Open Government Partnership que en este documento se identifica como Alianza para el Gobierno Abierto (AGA).

\section{Transparencia y gobierno abierto}

Al inicio de su primer periodo gubernamental, Barack Obama firma el memorándum Transparencia y Gobierno Abierto. En él se expresa el compromiso de crear un nivel de apertura gubernamental sin precedentes (Obama 2009) a partir de tres características: 1) el gobierno debe ser transparente, como característica que promueve la rendición de cuentas; 2) el gobierno debe ser participativo, como medio para incrementar la efectividad y calidad de sus decisiones aprovechando el conocimiento que se encuentra disperso entre su gente; 3) el gobierno debe ser colaborativo, usando herramientas, sistemas y métodos innovadores que promuevan el intercambio y retroalimentación en todos los niveles y considerando las organizaciones sin fines de lucro, negocios e individuos del sector privado. Hasta aquí la descripción de un deseo y sus elementos. Por este medio se instruye a diversas oficinas al interior de la Presidencia para que, en un plazo de 120 días, lleven estos postulados a la práctica.

\section{Directriz para el gobierno abierto}

En diciembre de 2009, el director de la Oficina de Administración y Presupuesto de la Presidencia de Estados Unidos (OMB, por sus siglas en inglés), Peter R. Orszag, envió el M-10-06². Ahí los pilares del gobierno abierto son la transparencia, la participación y la colaboración (Orszag 2009).

2 El M-10-06 tiene por título Open Government Directive. 
De acuerdo con este documento, son cuatro los pasos necesarios para crear un gobierno abierto: 1) publicar en Internet información gubernamental; 2) mejorar la calidad de la ya publicada; 3) crear e institucionalizar una cultura de gobierno abierto; 4) crear y difundir un marco para las políticas de gobierno abierto. Este documento atiende al contexto real existente en la administración pública norteamericana y, a partir de ahí, construye una ruta crítica para su implementación.

La publicación en Internet de la información del gobierno es concebida como una oportunidad para incrementar la rendición de cuentas, promover la participación informada y crear oportunidades para la economía ${ }^{3}$. De aquí, dos las actividades por hacer: 1) cada agencia debía publicar, en formato abierto, al menos tres bases de datos de alto valor y registrarlas en un sitio único que los concentraría $\left.{ }^{4} ; 2\right)$ cada agencia crearía un portal de gobierno abierto localizado en una dirección estandarizada (www. nnombre de la agencia].gov/open), donde existieran mecanismos de comunicación y retroalimentación con la ciudadanía, pudiera obtenerse información sobre qué otra información debiera publicarse y se conociera un Plan de Gobierno Abierto particular de la agencia.

Para mejorar la calidad de la información gubernamental, segundo de los cuatro pasos ya descritos, se hace referencia a normatividad ya existente y el necesario acompañamiento de la $\mathrm{OMB}^{5}$. En este paso también se definen actividades específicas sujetas a un tiempo para su implementación: 1) designar a un funcionario de alto nivel como el responsable de la calidad y la objetividad de la información; y 2) establecer un marco básico o guía mínima referida a la calidad de la información del gasto y publicar

3 Las referencias a las normatividades relacionadas sirven como soporte sobre cómo y qué debe publicarse: la Federal Records Act, la Freedom of Information Act, la circular OMB A-I 30 para la administración de la información gubernamental.

4 Se trata del sitio www.data.gov Al mes de junio de 2015, en este lugar pueden consultarse más de 140.000 bases de datos agrupados en catorce temas: agricultura, negocios, clima, consumo, ecosistemas, educación, energía, finanza, salud, gobierno local, manufactura, océano, seguridad pública, ciencia. El mismo sitio se declara como una fuente para el hackeo cívico, los emprendedores tecnológicos, los científicos y todo tipo de desarrolladores.

5 Las referencias específicas con la Information Quality Act y lo que podría traducirse como "Guías para asegurar y maximizar la calidad, objetividad, utilidad e integridad de la información diseminada por las agencias federales". Esto último es efectivo desde octubre de 2001 . 
información del gasto ${ }^{6}$.

Crear e institucionalizar la cultura del gobierno abierto se define como un trabajo multidisciplinar al interior de las agencias gubernamentales. En el documento se establece una ruta crítica para hacerlo, para gestionar el cambio de la cultura organizacional. Un último paso es el de la creación y difusión de un marco para las políticas de gobierno abierto. Aquí el asunto se aborda de manera más general: se establece que a los 120 días la Oficina de Asuntos de Información y Regulación habrá revisado cuáles de los trámites existentes podrán ser automatizados e integrados a una plataforma tecnológica. Y hasta aquí el primero de los dos apartados del documento.

El segundo de los apartados del M-10-06, describe los contenidos mínimos de un Plan de Acción que, de acuerdo con el documento, deben incorporar el parecer de un funcionario senior del área jurídica, tecnológica o policy y el público en general o expertos en apertura gubernamental. La estructura propuesta debe mejorarse, señala el documento, con la incorporación del ciudadano y los servidores públicos durante el proceso.

Los componentes del Plan coinciden con los pilares del gobierno abierto: Para el aspecto de la transparencia, deberá establecerse cómo la agencia la incrementará identificando la que más valoran sus usuarios y poniéndola a disposición para ser aprovechada por el ciudadano común. Aquí, el valor de la información está determinado por la posibilidad de que su uso incremente la rendición de cuentas (accountability) y la capacidad de respuesta (responsiveness) de la agencia. En cuanto a la participación, el Plan deberá describir la revisión que haga la agencia para encontrar áreas de oportunidad y, cuando se requiera, el rediseño de mecanismos de retroalimentación del público. En el aspecto de la colaboración, la agencia deberá detallar: 1) cómo podría mejorar la comunicación con personas fuera de la agencia mediante el uso de las plataformas tecnológicas; 2) cómo establecerá algún espacio (un sitio web) donde puedan conocerse actividades existentes de colaboración y aprender de ellas; y 3) cómo establecerá métodos innovadores (competencias, premios) para incentivar

6 Particularmente en lo que se requería en virtud de dos normativas prácticamente simultáneas al documento que se está refiriendo: En febrero de 2003, en un contexto de crisis económica, el Congreso Norteamericano aprobó un paquete especial de estímulos económicos llamado The American Recovery and Reivestment Act por el que se aplicarían alrededor de 800 millardos de dólares; en septiembre de 2006 se expidió la Federal Funding Accountability and Transparency Act Subaward Reporting System cuyo objetivo es el poder dar seguimiento a los contratos y apoyos otorgados por el gobierno estadounidense superiores a US \$25.000. Estas dos normativas son las incorporadas a los 120 días. 
la colaboración. Finalmente, el Plan de Acción deberá contener una iniciativa insignia (flagship initiative), donde se deja a cada agencia la construcción de, al menos, una medida que contribuya en alguno de los pilares del gobierno abierto.

¿La experiencia norteamericana? A partir de una base general y la gestión del cambio, promueve el trabajo de sus organizaciones desde sus propias decisiones y considerando su realidad, sus capacidades, recursos y fines. Se promueve la iniciativa y la competencia.

\section{Alianza para el Gobierno Abierto}

En septiembre de 2010, en su discurso a la Asamblea de las Naciones Unidas, el presidente norteamericano Obama habló de la complicada situación económica mundial, la amenaza que significaban Al Qaeda y los talibanes, la añoranza de la paz entre israelís y palestinos. En el desahogo de las prioridades de la agenda, en un punto señaló:

Como dije el año pasado, cada país seguirá el camino enraizado en la cultura de su pueblo. Sin embargo, la experiencia nos enseña que el progreso de la humanidad descansa en la economía abierta, la sociedad abierta, el gobierno abierto (Obama 2010. Traducción propia).

Otra manera de frasear los principios y valores liberal-democráticos, si se lee con atención. Ahí mismo, propuso regresar al año siguiente con propuestas específicas para promover la transparencia, combatir la corrupción, avivar el compromiso ciudadano y apalancar el uso de la tecnología para fortalecer los cimientos de la libertad en los países7.

Consecuencia de lo anterior, en 2011 se constituye la Alianza para el Gobierno Abierto (AGA) como iniciativa multilateral que apoya los esfuerzos nacionales para promover la transparencia, combatir la corrupción, fortalecer la rendición de cuentas y empoderar al ciudadano (The White House. Office of the Press Secretary 2011). La presentación oficial en Nueva York estuvo a cargo del propio presidente Obama y su homóloga de Brasil, Dilma Rousseff. El documento fundacional, Open Government Declaration, establece los siguientes compromisos: 1)

7 Traducción libre de otra parte del discurso ya referido en la nota anterior. Textualmente dice: "And when we gather back here next year, we should bring specific commitments to promote transparency; to fight corruption; to energize civic engagement; and to leverage new technologies so that we strengthen the foundation of freedom in our own countries, while living up to ideals that can light the world'. 
incrementar la disponibilidad de la información gubernamental; 2) apoyar la participación cívica; 3) implementar altos estándares de integridad profesional en el servicio público; 4) incrementar el acceso a nuevas tecnologías para la apertura y la rendición de cuentas (Open Government Partnership 2011).

De 2011 a 2015, el número de países miembros ha pasado de 8 a 65. El número es importante no solo por su obvio incremento sino porque cada uno debió satisfacer un procedimiento ${ }^{8}$, cumplir con algunos requerimientos ${ }^{9} \mathrm{y}$ pagar una cuota anual. AGA es el ente promotor, articulador y, de alguna manera, validador de los esfuerzos nacionales de gobierno abierto.

Pues bien, ¿`cuál es el esquema de gobierno abierto que AGA promueve? Para decirlo en una frase, no hay una respuesta definitiva para esto (Transparency and Accountability Initiative 2015). Ofrecen una definición de trabajo (working definition) que es, en realidad, la propuesta por Nathaniel Heller (2012) en el blog de la organización Global Integrity. Una base general y cada país le da sentido. En la siguiente tabla los elementos.

8 Son tres los pasos a seguir por el país que quiera pertenecer a AGA: 1) el gobierno debe demostrar un nivel mínimo de compromisos en cuatro áreas estratégicas (se describen a continuación); 2) enviar una carta de intención; 3) identificar a la unidad administrativa o titular de área que se hará cargo del desarrollo del Plan de Acción.

9 Estos son de dos tipos: Por una parte se definen a partir de las cuatro áreas estratégicas en las que se debe demostrar un compromiso mínimo: 1) transparencia fiscal, de acuerdo con la información del Open Budget Index; 2) acceso a la información, de acuerdo con la valoración del proyecto rigth2info.org; 3) normatividad para transparentar el ingreso de los servidores públicos designados y electos y 4) la participación de la ciudadanía en procesos de política pública, donde se emplea el The 2012 Economist Intelligence Unit Democracy Index. El segundo grupo de requerimientos se componen por las actividades a desarrollar: 1) el desarrollo de un plan de acción a través de un proceso abierto y plural que contenga compromisos específicos y medibles; 2) el desarrollo de un plan de implementación; 3) la preparación de una auto-supervisión anual; 4) la participación en el denominado Independent Reporting Mechanism que elabora reportes bianuales de avances. 


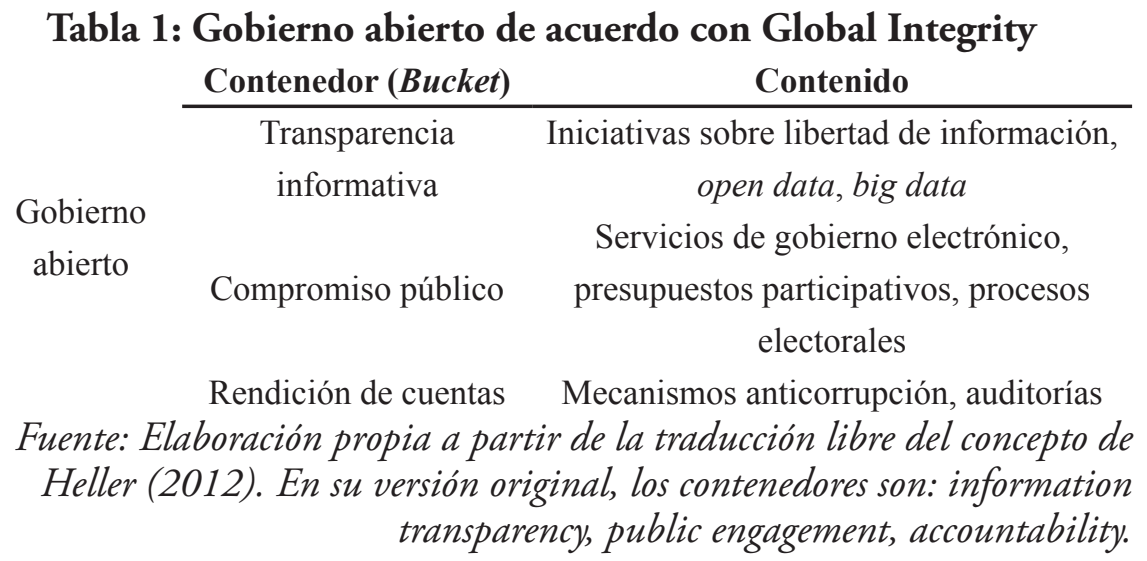

Heller (2012) enfatiza que los componentes por sí solos no son gobierno abierto, se requiere la combinación de ellos. Sobre el uso de la tecnología expresamente señala que no es un requerimiento para la existencia del gobierno abierto.

¿La experiencia de AGA? Una base general que promueve la iniciativa y la competencia. Cada país, considerando su realidad, sus capacidades y recursos, le da sentido a la metáfora del gobierno abierto.

\section{MÉXICO: EL IMPULSO DE UN LABORATORIO SUBNACIONAL}

La dinámica internacional se ha llevado a la práctica en distintos países con diferentes matices. Para el caso particular, para observar cómo esto sucede, se describe y considera la experiencia mexicana a partir de la experiencia impulsada por el órgano rector nacional en la materia.

Formalmente, no hay un camino establecido para que una entidad subnacional pertenezca a la AGA (Open Government Partnership 2015). Se alienta, sin embargo, su integración como parte de los compromisos nacionales. En México se ha emprendido un esfuerzo importante y sin precedentes para llevar el llamado gobierno abierto al ámbito subnacional. Se coordina desde el Instituto Nacional de Acceso a la Información Pública (INAI) y es, sin duda, una experiencia de la que podrá (y deberá) aprenderse al mismo tiempo que valorar sus consecuencias.

Desde la perspectiva de las políticas públicas, la iniciativa de llevar al orden subnacional el tema generó (queriéndolo o no) un interesante laboratorio cuyo conocimiento y análisis es importante para el entendimiento del tema en lo específico y para la comprensión del funcionamiento del federalismo en la implementación de una política diseñada desde el centro. 
El punto de origen se ubica en 2014, cuando el INAI ${ }^{10}$ y dos comisiones especializadas de la Conferencia Mexicana para el Acceso a la Información Pública (COMAIP'11) desarrollaron una estrategia para avanzar en la consolidación de un Estado Abierto ${ }^{12}$ (IFAI-COMAIP 2015). Parte de ello derivó en la organización de ejercicios locales a implementarse en dos etapas semestrales, a lo largo de 2015 , en al menos doce entidades federativas.

\section{Consecuentes con la estrategia definida,}

[Se] realizó un diagnóstico de cada entidad federativa en donde se analizaron los temas principales de su agenda pública e identificaron actores sociales, organismos garantes, instituciones gubernamentales; proyectos y logros en materia de transparencia, rendición de cuentas y participación ciudadana; y los resultados de la métrica de transparencia 2014 (IFAI-COMAIP 2015).

Además, se envió un cuestionario con once preguntas para recabar datos relacionados con actores, contexto político, problemáticas identificadas y su alineación con la agenda de la AGA. Posterior a esto, se desarrolló una serie de indicadores (a partir de 14 criterios, dentro de ellos de dos a tres supuestos con diferentes grados de avance y a cada uno) y se le asignó una puntuación siendo que a mayor número obtenido, mayor las fortalezas demostradas para el programa ${ }^{13}$. Del cruce de esta información, se repartieron las entidades participantes en dos grupos o bloques, siendo los colocados en el primero las de "mejores condiciones para realizar los

10 Entonces será el Instituto Federal de Acceso a la Información y Protección de Datos (IFAI). En el texto se empleará su nombre actual y sus siglas INAI excepto en las citas de documentación realizadas bajo la anterior denominación.

11 Es, para tener una rápida definición, un espacio de diálogo y trabajo que reúne a todos los órganos garantes del derecho de acceso a la información de los estados, entidades que componen la República.

12 Nótese que, para el caso particular, no trata del gobierno abierto sino un concepto con pretensiones más amplias: el Estado abierto.

13 La nota 2 del documento en cita (IFAI-COMAIP 2015) dice: "Es importante señalar que, si bien Jalisco pudo haber formado parte del primer bloque de ejercicios locales por haber obtenido una mayor puntuación con respecto a otras entidades federativas, éste manifestó su interés expreso por formar parte del segundo bloque de ejercicios locales, razón por la cual se consideró a Puebla para el primer bloque" 
ejercicios en primera instancia” (IFAI-COMAIP 2015) ${ }^{14}$.

En marzo de 2015, en las instalaciones del INAI, se celebró el evento Gobierno Abierto. Co-creación desde lo Local, donde tuvo verificativo una firma protocolaria del compromiso que asumían las entidades subnacionales en cuanto a los ejercicios locales y se presentó la propuesta de un esquema particular descrito a continuación.

\section{Modelo de gobierno abierto para gobiernos subnacionales}

En el marco del evento de marzo de 2015, el INAI capacitó a los representantes de las entidades federativas. Ahí expusieron que el gobierno abierto es:

$[\mathrm{U}] \mathrm{n}$ modelo de gestión que incorpora principios, políticas o acciones de transparencia, acceso a la información, rendición de cuentas, participación ciudadana y co-creación gubernamental, apoyados en las TIC y orientadas a lograr niveles de apertura y colaboración que permitan generar beneficios colectivos (IFAI 2015a).

En este material se identifican cuatro componentes del gobierno abierto. Un mes después, habían cambiado.

14 En retrospectiva es importante señalar que, ignorando sus propios criterios, colocaron a Puebla dentro del primer grupo para la implementación sustituyendo a Jalisco quien expresamente solicitó estar en el segundo de los grupos (ver nota previa). Esto es significativo a la luz de lo que después aconteció y que se describe en el apartado siguiente. 


\title{
Tabla 2: Evolución de componente en el modelo INAI ${ }^{15}$
}

\author{
Componentes del gobierno abierto
}

(según el INAI, propuesta para gobiernos subnacionales)

En la capacitación a gobiernos

subnacionales, marzo de 2015
En documento para consulta pública, abril de 2015

Acceso a la información

Transparencia inteligente

Rendición de cuentas

Participación ciudadana efectiva

Colaboración y co-creación gubernamental
Transparencia proactiva

Rendición de cuentas

Participación ciudadana

Co-creación

Innovación

Fuente: Elaboración propia a partir de la información elaborada por el ahora INAI (2015a, 2015b).

Independientemente de que el contenido de los elementos merece un estudio de mayor calado, su variación (en número y denominación) es importante si se considera la discusión interior que debió darse y cómo podría incidir esto en el comportamiento del implementador: cómo distinguir, por ejemplo, si lo emprendido es transparencia inteligente o proactiva cuando, en su trabajo cotidiano, esos adjetivos no se emplean. Igualmente importante es comparar los elementos del modelo INAIGobiernos subnacionales con los que hay en otras referencias: dada su más

15 Para abril de 2015, el documento sometido a consulta pública explicitaba de mejor manera cada uno de los componentes: acceso a la información, como derecho humano reconocido en tratados internacionales como la Declaración Universal de Derechos Humanos y la Convención Americana sobre Derechos Humanos; transparencia proactiva. La publicación de información adicional a la establecida en la Ley General de Transparencia y Acceso a la Información Pública orientada a la generación de conocimiento público útil capaz de resolver problemas y hacer más efectiva la toma de decisiones de autoridades y ciudadanos; rendición de cuentas. En un sentido amplio, es el proceso de evaluación del desempeño de las autoridades por parte de la ciudadanía; participación ciudadana, La posibilidad de incidencia social en los procesos de adopción de las decisiones colectivas; co-creación. Lograr que el ciclo de la política pública sea resultado de un trabajo compartido entre la sociedad y las autoridades públicas, desde la identificación de problemas, generación de conocimiento público y la propuesta de alternativas de solución, hasta la implementación de acciones y su evaluación; innovación, mediante el uso de las tecnologías de la información y comunicaciones (TIC), incluyendo también la cívica y la social. 
alta complejidad, el subnacional demanda mayor detalle y los requisitos por satisfacer son mayores. Sin perder la línea del argumento ¿qué implicaría para el implementador subnacional trabajar con un concepto más complejo?

Al momento (agosto de 2015) no se ha publicado una versión definitiva del modelo, producto en parte de la consulta pública realizada por el propio INAI. Como ejercicio, la búsqueda de un concepto para el gobierno abierto es importante: determinar su contenido mínimo es lo que ofrece la oportunidad de instrumentar una política pública coherente.

\section{Algunas experiencias y hallazgos preliminares}

Lo hasta ahora acumulado a lo largo de 2015 en el orden subnacional mexicano es ya de alto valor para entender el gobierno abierto. Algunos ejemplos: en Coahuila el componente de la participación ciudadana ha obligado a una adecuación de la propuesta inicial; en Veracruz mostraron iniciativa al crear una figura facilitadora dentro de cuerpo colegiado implementador del gobierno abierto; Morelos ha manifestado que requirieron de contratar asesores externos; en Puebla el asunto ya no pudo avanzar a partir de los parámetros seńalados.

Ninguna de estas experiencias descalifican lo emprendido, pero la señal es clara: el contexto se impone para dotar de significado a la metáfora.

En una de las actas de la experiencia en Veracruz puede leerse: “[...] el Dr. Felipe Hevia de la Jara, en uno de la voz manifestó: tomando en cuenta que esto es un machote para los otros estados y que se tiene que adaptar [...] propone separar lo sustantivo del Secretariado de lo administrativo [...]" (Veracruz- Secretariado Técnico Local 2015: 2).

Estas adecuaciones hay que enfatizarlas, saber que se puede. El contexto.

Sobre el caso Puebla se ha dicho: "Si llegaran a desarrollar una estrategia de gobierno abierto estaría avalada por nadie; sería un ejercicio entre cuates para aplaudirle a los cuates" (Aroche 2015). Sin filias ni fobias debiera pensarse en el gobierno abierto no para avalar algo sino para, en un aspecto amplio, mejorar la comunicación entre gobiernos y gobernantes. Se trata de sumar, no restar. Propiciar la iniciativa y promover la creatividad en la implementación mediante el conocimiento del contexto y no monopolizar el tema para descalificar a quienes pueden hacer algo diferente.

¿La experiencia del INAI hasta ese momento? Pues que de manera natural, las organizaciones se abren camino para adaptar un concepto 
de alta complejidad. Parten, por cierto, de su contexto: su realidad, sus capacidades y recursos.

\section{Un nuevo modelo para 2016}

A partir de la experiencia de 2015, hacia finales de ese año, el INAI promovió la construcción de un Modelo de Gobierno Abierto. Al final, este modelo consideró la consulta hecha vía electrónica a poco más de 200 interesados y especialistas en el tema provenientes de la academia, de la sociedad civil organizada, de los organismos garantes locales, así como de instituciones públicas federales y locales no solo de México, sino de especialistas de Brasil, Chile, España, Estados Unidos y Perú, tres mesas temáticas de discusión (transparencia, participación ciudadana e innovación) que contaron con la participación de varias decenas de especialistas provenientes del sector público, la academia y la sociedad civil organizada. La base teórica de ese ejercicio fue sometida a consideración del Sistema Nacional de Transparencia (colectivo que coordina las representaciones de organismos relacionados con esa temática) y fue aprobada para su implementación a inicios de 2016.

De este ejercicio destaca una propuesta conceptual: el gobierno abierto es un esquema de gestión y producción de políticas públicas orientado a la atención y la solución colaborativa de los problemas públicos con base en colegiados plurales y, en cuyo trabajo, convergen la transparencia y la participación ciudadana como criterios básicos, en un ambiente de rendición de cuentas e innovación social. Junto con la definición, se desarrolló un esquema para la capacitación e implementación, considerando tanto al gobierno como a la ciudadanía organizada. A partir de junio de 2016, comenzó su práctica por lo que habrá que esperar para conocer cómo es que esta propuesta engarza con la realidad.

\section{DE METÁFORAS Y POLÍTICAS PÚBLICAS}

Una breve referencia de algo que ya se ha visto: cuando en México comenzaba a hablarse de la transparencia, allá iniciando la década pasada, no hubo mejor manera de hacerlo que echando mano de algunas metáforas: "poner al gobierno en una caja de cristal" o "entrar hasta la cocina del gobierno". Cómo podría ser de otra manera si el término transparencia es, en realidad, la cualidad de algún material o cuerpo por el que se puede ver claramente. Transparencia aplicado a lo gubernamental es, pues, también una metáfora (Christensen y Cornelissen 2015). 
La metáfora facilita la comprensión de lo complejo: si alguien observa a su gobierno dentro de una caja de cristal, no hay secretos; entrar hasta la cocina, es poder compartir un espacio que, por diversas razones, había sido reservado solo para unos pocos. Un gobierno transparente es aquel que no tiene lugares opacos, donde la luz pasa sin dificultad.

El asunto se complica, sin embargo, cuando en la realidad no hay una caja de cristal ni una cocina como tal: los gobiernos no son materia por el que la luz pueda pasar sino organizaciones y personas regidas por normas y restringidas por presupuestos.

El uso de la metáfora en una reforma y en una política es válido y hasta necesario, dice Arellano Gault (2008). Sin embargo, agrega, es necesario avanzar en al menos dos sentidos:

$[\mathrm{U}]$ no, sacando a flote los supuestos argumentativos y la solidez teórica y práctica de los supuestos que sostienen a la metáfora [...] Segundo, relacionar las especificidades de los aparatos administrativos contemporáneos para comprender cuáles pueden ser las mejores estrategias para avanzar en la agenda (Arellano Gault 2008: 264-265).

Y es que dejar el asunto a nivel de metáfora confunde e imposibilita un avance concreto.

Ahora, en diversos foros y discusiones, la agenda dominante presenta una nueva idea que, parece, llegó para quedarse: el gobierno abierto.

¿Qué es el gobierno abierto? Depende quién y cuándo responda la pregunta.

Esta situación, que debiera preocupar a más de un implementador (pues ¿a cuál de todas las definiciones debiera destinar tiempo, dinero y esfuerzo?), realza la importancia de un trabajo permanente que explore similitudes y diferencias entre las diversas manifestaciones del gobierno abierto, sus requerimientos y exigencias. Pone de manifiesto, también, la delgada línea existente entre el desarrollo de un concepto para la implementación de alguna política pública frente a un concepto para el monopolio de la política pública. Sobre esto último, elaboro un poco más.

La caja de cristal, entrar hasta la cocina: dos de los muchos ejemplos que podrían citarse. La comunicación se nos facilita gracias a las metáforas. Explorarlas como fenómeno lingüístico aplicado a la realidad, sin embargo, es un campo cuya extensión no va más allá de la década de los años setenta 
del siglo pasado (Camp 2005). Por esto, y la poca producción académica y analítica al respecto, es que el tema suele pasar desapercibido.

De las muchas aristas por explorarse, nos quedamos con una: el aspecto práctico de la metáfora. Éste está dado, de acuerdo con Stern (2000), por la convivencia entre la semántica (lo literario de la expresión, dicho en lenguaje llano) con el contexto en el que se emplea. La identificación de esta convivencia es lo que vuelve el trabajo de Stern ${ }^{16}$ una referencia pues, previo a él, la metáfora solo se estudiaba como semántica, creyendo que su significado estaba naturalmente dado (Camp 2005).

Así, por más obvio que parezca qué debe entenderse por gobierno abierto, la verdad es que no es algo dado de manera natural o que pueda encontrarse de manera directa. Alguien debe, partiendo de alguna referencia, delinear los parámetros de convivencia: el trabajo a favor de un entendimiento convencional y estable de una metáfora abona a una interacción eficiente (Molek-Kozakowska 2014). La referencia se encuentra en el contexto, por lo que un gobierno abierto para Espańa y los españoles, por ejemplo, puede no ser lo mismo que en México y los mexicanos. Y la misma lógica aplicaría a lo subnacional: mientras que en un contexto político-gubernamental rígido (por llamarle de alguna manera) el gobierno abierto pudiera ser la oportunidad para dialogar con diferentes actores, en otro mucho más flexible eso queda bastante corto.

Pero, si el entendimiento de una metáfora depende del contexto y cada contexto es diferente, ¿̇no estaríamos frente a un sinnúmero de gobierno(s) abierto(s)? Aun cuando la respuesta pareciera ser en principio un sí, la propagación y vigencia de principios y valores liberal-democráticos ha hecho, de la comunidad global, una más homogénea de lo que pudiera

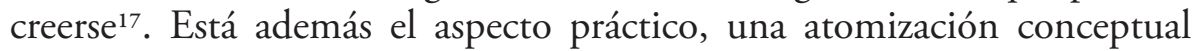
ad infinitum dificultaría la comunicación entre pares. Para decirlo en una frase: alrededor del gobierno abierto, asunto que nos ocupa, la construcción de una comunidad no solo es deseable sino posible al considerar, como referencia, un contexto liberal-democrático que enmarque la metáfora.

16 El trabajo del profesor Stern es, por supuesto, mucho más amplio que el texto aquí citado. Una buena parte puede conocerse en su página del Departamento de Filosofía de la Universidad de Chicago en http://philosophy.uchicago.edu/faculty/stern.html

17 Dada la homologación alrededor del ideal liberal-democrático, debiera haber un número finito de esquemas de Gobierno Abierto. La discusión sobre los principios y valores liberal-democráticos es, por supuesto, muy amplia y no es ajena a debate. Abordarlo en este trabajo en la medida requerida, lamentablemente, es imposible. 
El siguiente paso requiere la identificación de quién y cómo le da contenido a la metáfora. Desde esta perspectiva hay una doble alternativa: tener un concepto para la implementación de alguna política pública o tener un concepto para el monopolio de la política pública. Esta diferencia se percibe en la medida que se privilegien o no principios y valores liberaldemocráticos, como el respeto a la pluralidad, la persona, la propiedad, la vida, por citar algunos.

Un concepto para la implementación de alguna política pública es el que define elementos básicos suficientes (un piso mínimo, usando otra metáfora) para que el implementador oriente sus propias decisiones a partir de su realidad, sus capacidades, recursos y fines; este concepto suele promover tanto la iniciativa como la competencia. Un concepto para el monopolio de la política pública, por el contrario, es aquel que restringe la pluralidad, ignora las capacidades de los implementadores, su realidad y recursos y tiene mecanismos que incentiva la observancia acrítica de la instrucción al tiempo que castiga (o desprecia) la iniciativa y la competencia.

Advertido lo anterior, de regreso al punto de origen: ¿qué es el gobierno abierto? Exploremos la literatura especializada.

Para Insulza se trata de una "política pública que agrupa los conceptos de transparencia, participación y colaboración” (2012: 9). Éste es un concepto agregativo de aparente sencillez construído a partir de los elementos que le componen. Manuel Villoria Mendieta, por su parte, opta por una definición que llama normativamente maximalista: un gobierno abierto, dice, es "aquel que entabla una constante conversación con los ciudadanos con el fin de oir lo que ellos dicen y solicitan, que toma decisiones basadas en sus necesidades y preferencias, que facilita la colaboración de los ciudadanos y los funcionarios" (2012: 71). Para Cruz-Rubio, más o menos en la misma línea que la anterior, se trata de "una filosofía político administrativa y nuevo paradigma o modelo de interacción sociopolítica" (2015: 39). También se le conceptualiza como la expresión de principios que se manifiestan en la mejora de los niveles de transparencia y acceso a la información, una mayor facilidad para la participación ciudadana y la generación de espacios de colaboración entre diversos actores (Ramírez Alujas y Dassen 2012).

Se ha planteado, también, como una evolución de lo que por más de una década se ha denominado como Gobierno Electrónico y su conjunción con otras "tres dimensiones o pilares fundamentales" (Naser y Ramírez Alujas 2014: 6): 1) la transparencia en la acción, procesos y datos del gobierno, 2) la colaboración al interior del Gobierno y con las personas 
que permita generar nuevas ideas para resolver problemas sociales; y 3) la participación que buscar implicar de forma activa y real a los ciudadanos en la formulación y ejecución de políticas. En la línea evolutiva, César Calderón lo plantea como

[...] una evolución de nuestro sistema democrático de convivencia y valores basada en el establecimiento de mecanismos para la transparencia de los gobiernos, así como de espacios permanentes de colaboración y participación de los ciudadanos más allá del ejercicio del derecho de sufragio" (2012: 27).

Para Nicandro Cruz-Rubio es "un modelo relacional que se corresponde con un modelo de democracia más agregativo, que da (busca dar), prioridad a la representatividad y los procesos y cuya orientación es la de conformar un gobierno (más) accesible, transparente y receptivo" (2015: 39). Él mismo avanza conceptualmente al decir lo que no es: gobierno abierto no es gobierno electrónico; gobierno abierto es más que gobernanza electrónica; gobierno abierto no es equivalente a datos abiertos (Cruz-Rubio 2015). El recorrido conceptual puede seguir.

El punto a destacar es que no hay un acuerdo amplio ni más o menos uniforme. Como consecuencia, entre quienes hablan de gobierno abierto es normal existan tantas aproximaciones como sus lecturas y experiencias les provoque. Esto, si bien resulta enriquecedor en el orden de las ideas, dificulta la construcción de una comunidad, alienta la imposición de un término (el concepto para el monopolio de la política pública) y, por lo mismo, dificulta su ejercicio.

Desde la perspectiva de la política pública, la pregunta es: ¿a cuál de todas las definiciones se le debe destinar tiempo, dinero y esfuerzo? Aquí la respuesta pudiera no estar tanto en la discusión teórica sino en la observación de la experiencia. Ahí, en la realidad, es que las organizaciones y las personas se han enfrentado a la normatividad y las restricciones presupuestales y de todo tipo queriéndole dar contenido a la metáfora. Lo han hecho, sabiéndolo o no, atendiendo a un contexto.

\section{Sobre la pertinencia de (algún) esquema}

Un ejercicio necesario es conocer las similitudes y diferencias de cada concepto. Encontrar los puntos coincidentes y buscar las razones de las diferencias. 
Cada realidad, su contexto, ha moldeado el concepto. ¿El mensaje? Pues que debe considerarse la implementación como algo central. No hacerlo así, mantener la postura de que el contenido de la metáfora está dado de manera natural, implica un alto riesgo de fracaso para los diseñadores, los implementadores y, en general, la población que no se verá beneficiada por un esquema de gobierno abierto.

Una aproximación desde la disciplina de las políticas públicas permite hacerse cargo de la nada sencilla tarea de identificar la información con la que se cuenta, analizarla y ofrecer diferentes líneas de acción para mejorar la calidad de la decisión tomada, sea en el momento en que se toma o mientras se realiza (Aguilar Villanueva 2006). En esta línea, regresar a lo básico y adoptar un concepto agregativo (de sencillez aparente) como el de Insulza, no es un retroceso. Aún ahí hay mucho por hacer. Veámoslo con mayor detenimiento.

En dos de los componentes del concepto de Insulza, la transparencia y la participación, el asunto ya es complejo. Atendiendo a sus objetivos, resulta que no hay solo un tipo de transparencia, sino cinco (Peña 2009: 32): la que combate la corrupción, la que promueve el activismo cívico como medio para controlar el poder, la que propicia mejores servicios públicos, la que genera confianza incidiendo favorablemente en la inversión, la que legitima a los gobiernos. Cada tipo tiene sus estrategias para volverse una realidad.

Algo similar ocurre con participación: desde finales de la década de los años sesenta, Sherry R. Arnstein (1969) propuso la metáfora de los ocho peldaños de la escalera de la participación ciudana para describir diferentes intensidades en esta práctica, a saber: la manipulación, la terapia (dos niveles de no-participación), la inconformidad, la consulta, el apaciguamiento, la colaboración, la delegación del poder y el control ciudadano. No hay, pues, una sola participación, sino varias clases de participación. La diferencia entre apaciguamiento y colaboración (en el trabajo de Arnstein) ilustra: en lo primero, se deja a los ciudadanos planear y proponer sin restricciones pero se mantiene el monopolio de la decisión sobre el valor, la pertinencia y la factibilidad; en lo segundo, por el contrario, se requiere una renegociación sobre la distribución del poder y en quién recae la calificación del valor, la pertinencia y la factibilidad.

Por si fuera poco, en el contexto latinoamericano -a diferencia de lo que se establece en literatura europea y norteamericana-, participar en una agrupación no necesariamente apuntala un activismo cívico (Klesner 
2007) como el que se necesitaría para el gobierno(s) abierto(s) ${ }^{18}$.

¿En cuál de las categorías podría ubicarse la realidad del lugar donde queremos implantar un gobierno abierto? Una honesta identificación de los elementos con que contamos facilitaría el diseño de estrategias y el tránsito a un modelo de gobierno abierto.

Lo anterior, en pocas líneas: cuando la transparencia con la que se cuenta es una que atiende prioritariamente a la legitimidad del gobierno (desatendiendo el activismo cívico como medio para controlar el poder) y la participación acostumbrada es con fines de consulta (cuarto peldaño en la escalera de Arnstein), la introducción de, por ejemplo, un ejercicio de open data enfrentará mayores barreras para su implementación dado el presupuesto (humano) necesario con el que no se cuenta. ¿Qué trabajo se requiere para impactar en estas variables?

Esta discusión nos lleva a reconocer que hablar (y acordar) sobre el gobierno abierto como concepto es apenas la mitad del camino. Resulta igual o más importante preguntarse cómo puede implementarse el gobierno abierto. Y esto requiere claridad en la gestión del cambio.

Desde la perspectiva de Naser y Ramírez Alujas (2014: 11), una serie de cambios son requeridos para que cualquier gobierno se integre a la idea de gobierno abierto: 1) cambio cultural, en cuanto a la comprensión de cómo y para qué el trabajo en las administraciones públicas; 2) cambio en los procesos en la administración pública, reingeniarlos para que sirvan al ciudadano y le sean cómodos; 3) cambio en la organización, para que la administración pública actúe bajo un modelo de red; 4) cambio en la forma de relación, para que se facilite el intercambio entre gobierno y gobernado. En la experiencia norteamericana puede identificarse, por ejemplo, lo de generar una cultura de gobierno abierto.

Diferentes maneras habrá para hacerlo, sin duda. La clave está en tener claro que nada se logra con voluntarismos normativos sino a través de la participación y el consenso de los involucrados (Richart Chacón 1993).

18 En su estudio basado en los casos de Argentina, Chile, México y Perú, Joseph L. Klesner concluye que, de acuerdo con lo que observa, la pertenencia a grupos profesionales, religiosos, deportivos y recreacionales no promueve activismo cívico. Esto, señala el autor, contradice la base de los argumentos de Putnam y el capital social. 


\section{REFLEXIONES FINALES}

El gobierno abierto es, en principio, el tácito reconocimiento de que la transparencia por sí sola no es suficiente.

Regresemos a lo básico, un concepto macro que oriente la política pública. El desarrollo de un modelo, como referencia ideal hacia donde los trabajos deben encaminarse, es compatible con el reconocimiento de diferentes esquemas donde cada organización muestre iniciativa a partir de su realidad.

Para el caso mexicano, la iniciativa mostrada por instancias subnacionales debe reconocerse e incentivarse. Valorar el esfuerzo genuino y no castigar la discrepancia entre la realidad y el modelo, pues tal vez la falla está en el modelo.

En el primer ejercicio del INAI con los estados, el desarrollado en 2015, se inscribieron 13 entidades federativas. De ahí, se instalaron 10 secretariados técnicos, es decir, el cuerpo plural que debía implementar los trabajos necesarios de acuerdo con los compromisos asumidos; al finalizar el ciclo, solo 3 lograron establecer un Plan de Acción dentro del tiempo originalmente contemplado ${ }^{19}$. De ellos aún no se tiene registro de cómo inciden en la vida de la población donde se encuentran. Es decir: la experiencia aún no muestra evidencia de algún beneficio medible al adoptar un esquema de gobierno abierto. ¿Cuál es el incentivo que tendrían los gobiernos, entonces, de participar.

Lo observado a la fecha hace suponer que los gobiernos participantes se han involucrado a partir del interés de la legitimidad que el tema, como algo nuevo y presente en la agenda como algo novedoso, parece ofrecer. La sociedad civil organizada local participa con menos intensidad con respecto a la sociedad civil organizada del centro del país, la cual participa a manera de facilitadores, capacitadores y promotores del tema.

La iniciativa del INAI por promover el gobierno abierto al orden subnacional debe, sin embargo, destacarse. Una forma de hacerlo es la de dar seguimiento al laboratorio de implementación que provocaron e insistir en que la importancia del gobierno abierto no está tanto en llegar a un concepto teórico universalmente aceptado sino en el beneficiar a la

19 Hacia mediados de 2016, el número de planes de acción elaborados y presentados se incrementó a 7. 
población desde la conjunción de la transparencia, la participación y la corresponsabilidad.

El gobierno abierto debe quedarse. Evolucionar a un Estado abierto, como se ha planteado, con aplicación a todas las organizaciones públicas.

A partir de una metáfora, acordemos un concepto que pueda llevarse a la práctica. Que no suceda como con la transparencia donde, tres lustros después, seguimos sin acordar qué debió lograrse con ella. Alejemos la discusión de los conceptos monopolizadores que restringen a los actores y castiga la iniciativa.

Algunos de los más importantes avances de la transparencia en México se gestaron en las entidades federativas y la experiencia ahí acumulada es esencial para comprender lo que funciona y no funciona en la implementación de una política pública. A la vuelta del tiempo, a fuerza de muchas voces desde el centro, pareciera que esto no es verdad. Sin embargo, lo es.

Sigamos en el tema del gobierno abierto más allá de la agenda internacional insistiendo en la natural correlación entre la transparencia y la participación en un contexto de corresponsabilidad entre gobiernos y gobernados como una práctica habitual, es algo bueno. 


\section{REFERENCIAS}

Aguilar Villanueva, L. F. (2006). Gobernanza y Gestión Pública. México: Fondo de Cultura Económica.

Arellano Gault, D. (2008). Transparencia y Organizaciones Gubernamentales. En Ackerman, J. M. (Ed.), Más Allá del Acceso a la Información. Transparencia, Rendición de Cuentas y Estado de Derecho. México: Siglo XXI, Instituto de Investigaciones Jurídicas de la UNAM, Universidad de Guadalajara, Cámara de Diputados, Centro Internacional de Estudios sobre la Transparencia y el Acceso a la Información.

Arnstein, S. R. (1969). A Ladder of Citizen Participation. Journal of the American Institute of Planners, 35 (4), 216-224.

Aroche, E. (2015). INAI Hace a un Lado a Puebla de las Acciones de Gobierno Abierto. Portal de Transparencia Con los Ojos Abiertos, 22 de junio. Disponible en http://conlosojosabiertos.org.mx/index. $\mathrm{php} /$ secciones/monitoreo/item/inai-hace-a-un-lado-a-puebla-de-lasacciones-de-gobierno-abierto [23-06-2015]

Calderón, C. (2012). Por Qué un Gobierno Abierto. En Concha, G. y A. Naser, A. (Eds.), El Desafio hacia el Gobierno Abierto en la Hora de la Igualdad. Santiago: Comisión Económica para América Latina y el Caribe.

Camp, E. (2005). Josef Stern, Metaphor in Context. Noûs, 39 (4), 715731.

Canciller del Ducado de Lancaster. (1993). Open Government. Disponible en https://www.gov.uk/government/uploads/system/uploads/ attachment_data/file/271975/2290.pdf [18-08-2015]

Christensen, L. y Cornelissen, J. (2015). Organizational Transparency as Myth and Metaphor. European Journal of Social Theory, 18 (2), 132149.

Cruz-Rubio, C. N. (2015). ¿Qué Es (y Qué No Es) el Gobierno Abierto? Una Discusión Conceptual. Eunomia. Revista en Cultura de la Legalidad, 8, 37-53. 
Heller, N. (2012). A Working Definition of "Open Government". Disponible en https://www.globalintegrity.org/posts/workingdefinition-opengov/ [19-08-2015]

IFAI. (2015a). Gobierno Abierto. Material utilizado en el evento Gobierno Abierto Co Creación desde lo Local. México: IFAI.

IFAI. (2015B). Modelo de Gobierno Abierto - Documento de consulta. México: IFAI.

IFAI-COMAIP. (20 de Enero de 2015). Metodología de Evaluación para la Selección de Ejercicios Locales. Disponible en http://inicio.ifai. org.mx/Ms_Transparencia/Aciones/Evento\%20COMAIP\%2016\%20 de\%20Enero/Evaluaci\%C3\%B3n.pdf [27-06-2015]

Insulza, J. M. (2012). Prólogo. En Hofmann, A., Ramírez Alujas, Á. y Bojorquez Pereznieto, J. (Eds.), La Promesa del Gobierno Abierto. México: ITAIP - Info DF.

Klesner, J. L. (2007). Social Capital and Political Participation in Latin America. Evidence from Argentina, Chile, Mexico and Peru. Latin American Research Review, 42 (2), 2-32.

Molek-Kozakowska, K. (2014). Coercive Metaphors in News Headlines: A Cognitive-Pragmatic Approach. Brno Studies in English, 40 (1), 149173.

Naser, A. y Ramírez Alujas, Á. (2014). Plan de Gobierno Abierto. Una Hoja de Ruta para los Gobiernos de la Región. Santiago: Naciones Unidas. Comisión Económica para América Latina y el Caribe.

Obama, B. (2009). Memorandum for the Heads of Executive Departments and Agencies. Subject: Transparency and Open GovernmentDisponible en https://www.whitehouse.gov/the_press_office/ TransparencyandOpenGovernment [23-06-2015]

Obama, B. (2010). President Obama's Address to the United Nations General Assembly, September 2010. Disponible en http://www.cfr. org/international-organizations-and-alliances/president-obamasaddress-united-nations-general-assembly-september-2010/p23000 [23-06-2015] 
Open Government Partnership. (2011). Open Government Declaration. Disponible en http://www.opengovpartnership.org/about/opengovernment-declaration [18-06-2015]

- (2015). Open Government Partnership. Obtenido de FAQs. Disponible en http://www.opengovpartnership.org/about/faqs [18-06-2015]

Orszag, P. R. (2009). Memmorandum for the Heads of Executive Departments and Agencies. Disponible en https://www.whitehouse. gov/sites/default/files/omb/assets/memoranda_2010/m10-06.pdf [1806-2015]

Peña, V. S. (2009). Fortalecer desde la Experiencia. Apuntes para Mejorar el Dseño Institucional que Involucre Organismos Estatales de Acceso a la Información Pública. Toluca: Instituto de Acceso a la Inofrmación del Estado de México.

Ramírez Alujas, Á. y Dassen, N. (2012). Gobierno Abierto: La Ruta hacia una Nueva Agenda de Reforma del Estado y Modernización de la Administración Pública en América Latina y el Caribe. En Dassen, N. y Cruz Vieyra, J. (Eds.), Gobierno Abierto yTtransparencia Focalizda. Tendencias y Desafíos para América Latina y el Caribe. Washington: Banco Interamericano de Desarrollo.

Richart Chacón, J. A. (1993). La Formación como Factor de la Modernización y de la Calidad de la Administración. En Ministerios para las Adminsitraciones Públicas, Modernización Administrativa y Formación. Madrid: Ministerio para las Administraciones Públicas.

Sandoval Almazán, R. (2013). La Larga Marcha del Gobierno Abierto. Teoría, Medición y Futuro. México: Instituto Nacional de Administración Pública.

Stern, J. (2000). Methaphor in Context. Boston: Massachusetts Institute of Technology.

The White House. Office of the Press Secretary. (2011). Fact Sheet: The Open Government Partnership: https://www.whitehouse.gov/thepress-office/2011/09/20/fact-sheet-open-government-partnership [23-06-2015]

Transparency and Accountability Initiative. (2015). The Open Gov Guide. 
Disponible en http://www.opengovguide.com/glossary [23-06-2015]

Valenzuela Mendoza, R. (2014). Gobierno Abierto en una Perspectiva Multinivel: Reflexiones entre la Razón Teórica y la Innovación ráctiva. Revista de Gestión Pública, III (1), 163-197.

Veracruz - Secretariado Técnico Local. (2015). Portal para el Ejericcio de Gobierno Abierto en el Estado de Veracruz. Primera Sesión Ordinaria del Secretariado Técnico Local. Disponible en http:// www.veracruzgobiernoabierto.com.mx/2015/06/25/primera-sesionordinaria-del-secretariado-tecnico-local/ [07-08-2015]

Villoria Mendieta, M. (2012). El Gobierno Abierto como subsistema de políticas: Una evaluación desde el Institucionalismo Discursivo. En Hofmann, A., Ramírez Alujas, Á. y Bojorquez Pereznieto, J., La Promesa del Gobierno Abierto. México: ITAIP - Info DF

Weir, S. y Beetham, D. (1999). Political Power and Democratic Control in Britain. Londres y Nueva York: Rouledge.

Recibido: 30-05-2016

Aceptación de la versión final: 15-06-2016 